\title{
On an internal multimodel control for nonlinear multivariable systems - A comparative study
}

\author{
Nahla Touati Karmani \\ Automatic Research \\ Laboratory, LA.R.A, \\ National Engineering \\ School of Tunis (ENIT), \\ Tunis El Manar University, \\ Tunis, Tunisia
}

\author{
Dhaou Soudani \\ Automatic Research \\ Laboratory, LA.R.A, \\ National Engineering \\ School of Tunis (ENIT), \\ Tunis El Manar University, \\ Tunis, Tunisia
}

\author{
Mongi Naceur \\ Automatic Research \\ Laboratory, LA.R.A, \\ National Engineering \\ School of Tunis (ENIT), \\ Tunis El Manar University, \\ Tunis, Tunisia
}

\author{
Mohamed Benrejeb \\ Automatic Research \\ Laboratory, LA.R.A, \\ National Engineering \\ School of Tunis (ENIT), \\ Tunis El Manar University, \\ Tunis, Tunisia
}

\begin{abstract}
An internal multimodel control designed for nonlinear multivariable systems, is proposed in this paper. This approach is based on the multi-modeling of nonlinear systems and the realization of a specific inversion of each model. A comparative study is presented between two structures of the internal multimodel control. The first one is based on switching models and the second on residues techniques as fusion method. The case of a second order nonlinear multivariable system shows the effectiveness of both structures.
\end{abstract}

Keywords—internal multimodel control; nonlinear multivariable systems; inverse model; switching models; residues techniques.

\section{INTRODUCTION}

Among the control structure of physical systems, the Internal Model Control (IMC) is one of the powerful control laws for open-loop stable plants [6]. It provides an open-loop framework for checking closed-loop stability and also highlights the inherent performance limitations due to model uncertainties, non-minimum phase plant characteristics and actuator constraints.

The IMC systems are characterized by a control device consisting of the controller and a simulation model of the process. The internal model loop computes the difference between the process and its model which represents the effect of disturbances and model mismatch.

The proposed controller in this structure is the inverse of the selected model, if it is realizable. This task is the main problem of the IMC approach due to the difficulty of direct model inversion for the majority of physical systems, which gives a structure generally unrealizable [8].

The IMC design procedure is quite extensive and diverse. It has been developed in many forms; these include singleinput-single-output (SISO) and multiple-input-multiple-output (MIMO) formulations, continuous and discrete-time design procedures, design procedures for unstable open-loop systems, for nonlinear systems and so forth.

Developments of nonlinear IMC (NIMC) have been proposed for continuous time systems and for a class of discrete-time systems [9].
In this context, several NIMC schemes have been proposed, such us proposing an analytical inverse of the nonlinear model based on a physical understanding of the plant [10], or by representing the nonlinear plant by a collection of local linear models. The global controller in this case is obtained by combining these controllers using a fuzzy system. This last scheme combines two approaches: the internal model and multimodel control.

The multimodel approach is extremely interesting when dealing with nonlinear complex systems. In this approach, the nonlinear plant is described by a combination of local linear models, each of which is valid in a particular operating region. First, local controllers for the local models are tuned. Next, the control actions of these local controllers are combined in the form of a global controller to be implemented on the nonlinear plant. Different methods are proposed to design the global controller such us switching between local controllers or by combining them (residues techniques).

Based on the NIMC and the multimodel representation of nonlinear systems, this paper proposes the Internal MultiModel Control (IMMC) designed for nonlinear multivariable systems. Two structures will be presented, the IMMC based on the switching and the residues techniques.

This paper is organized as follows: section II presents a brief review on the internal model control strategy. Section III is devoted to multimodel approach. Section IV presents the proposed structure for the internal multimodel control designed for nonlinear multivariable systems. In section $\mathrm{V}$, an illustrative example is considered to show the effectiveness of the proposed structures and to compare them. Finally, concluding remarks are drawn in Section VI.

\section{NONLINEAR MUlTiVARIABLE INTERNAL MODEL CONTROL}

Internal model control is considered as a robust control method for open-loop stable systems. The nonlinear internal model control structure designed for multivariable systems is composed of a stable nonlinear multivariable process model, and a feed-forward controller as shown in Fig. 1. [2].

The controller is obtained as the inverse of the plant model 


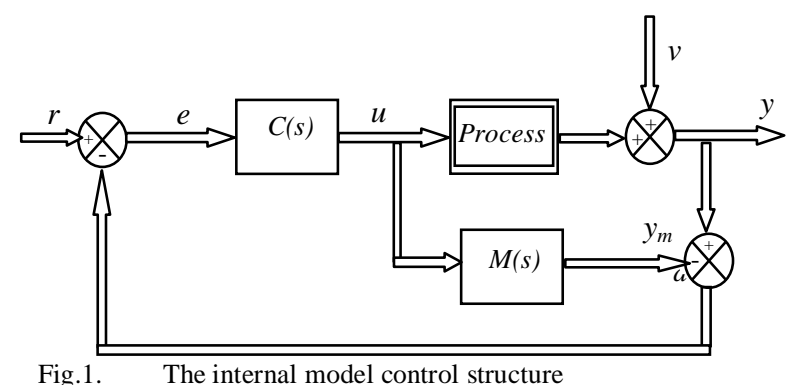

In the basic contiguration of the IMI tor multivariable systems, $M$ is the chosen model for the process, $C$ the controller and $v$ a vector of disturbance affecting the system. The manipulated $l$ input vector $u$ is introduced to both the process and its model. The difference $d$ between the process output vector $y$ of dimension $m$ and model output vector $y_{m}$, is compared to the reference vector $r$ to generate the controller input vector $e$. In this paper, we propose a fully actuated system. So, $l=m$.

The model inversion is the main problem of the IMC since the direct model inversion, for physical systems, gives a structure generally unrealizable.

The development of a nonlinear general approach of the IMC can raise serious difficulties, because of the complexity of the nonlinear systems, the absence of the mathematical inversion methods for the nonlinear models and consequently the difficulty of the design of a nonlinear internal model controller [1]. Multivariable linear internal model control is based on transfer matrix models, while nonlinear systems are usually described by nonlinear state-space models.

By using the inversion method proposed in [2], based on the gain matrix to realize an inverse model for the nominal model, we obtain the IMC controller shown in Fig. 2.

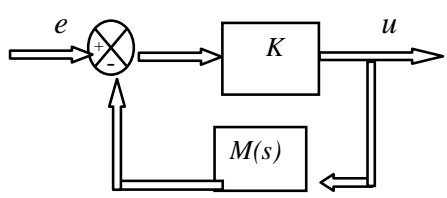

Fig.2. Generalized controller structure

\section{MULTIMODEL CONTROL}

The multimodel control represents a relatively new approach on nonlinear control strategies. It makes possible to represent nonlinear complex systems by using a set of simple linear models constituting a model's library, each of them is valid in a particular operating region. For each model, a suitable controller can be designed off-line [5].

The multimodel control can be perceived as a fusion of the partial command calculated from each model of this library or as a switching approach between these models.

The switching method consists on the choice of the nearest model to the process which leads to the least modeling error. The fusion method consists on evaluating the contribution of each partial command to the effective controller of the system.
It is based on calculating the residues which are frequently formulated by the geometric distance between the real output and the local models outputs [7]. The highest validity index is given to the system that gives the best estimation. In the opposite case the validity index is close to zero.

\section{MUltivariable INTERNAL MUltimOdEL CONTROL}

In this paper, an internal multimodel control is proposed for nonlinear multivariable systems as shown in Fig. 3. The first step consists on describing the nonlinear system to control by a model's library. The analysis and control of these linear models are easy and they can be exploited for an IMC structure. Identification or linearization around various operating points or convex polytopic transformation can be used to define these models.

The multimodel system describing the nonlinear multivariable system should be stable. In the proposed structure, the $M_{i}, i=1 \ldots n$, are linear state-space models describing the multivariable system. For each model $M_{i}$, a controller matrix $C_{i}$ is designed in order to ensure the nominal performances for the pair $\left(M_{i}, C_{i}\right) . C_{i}$ is the IMC controller obtained by using the inversion method as proposed in Fig. 2. It is integrated in the closed-loop configuration, as presented in Fig. 3. All local controllers are calculated instantly and simultaneously. $y$ and $u$ are the system output and input vectors. $\tilde{y}_{i}, \tilde{u}_{i}$ and $e_{i,} i=1 \ldots n$ present the output, input and controller input vectors respectively for each model $M_{i} . d$ is the modeling error, $K_{i}$ the gain matrices and $v$ the validation index.

Two methods are proposed in this paper, the first one is based on the switching technique: the model with the smallest error is chosen. After this operation, the correspondent control input $u$ is applied to the system. The second method is based on residues techniques.

The calculation of the controller to be applied to the real system depends, not only on the partial controllers, related to the library's models, but also of the validities resulting from the residues which represent errors between system and models outputs.

\section{A. System description}

Let's consider a nonlinear multivariable system described by the following state space equation

Where

$$
\left\{\begin{array}{l}
\dot{x}=f(x, u, t) \\
y=g(x, u, t)
\end{array}\right.
$$

- $x$ is the system state vector, $x \in R^{m}$;

- $y$ is the output vector, $y \in R^{m}$;

- And $u$ is the input vector, $u \in R^{m}$.

This system can be represented by a library of $n$ linear models $M_{i}$, where each model can be described by the following state space equations

$$
\left\{\begin{array}{l}
\dot{x}_{l}=f_{i}\left(x_{i}, \tilde{u}_{i}, t\right) \\
\tilde{y}_{i}=g_{i}\left(x_{i}, \tilde{u}_{i}, t\right)
\end{array}\right.
$$




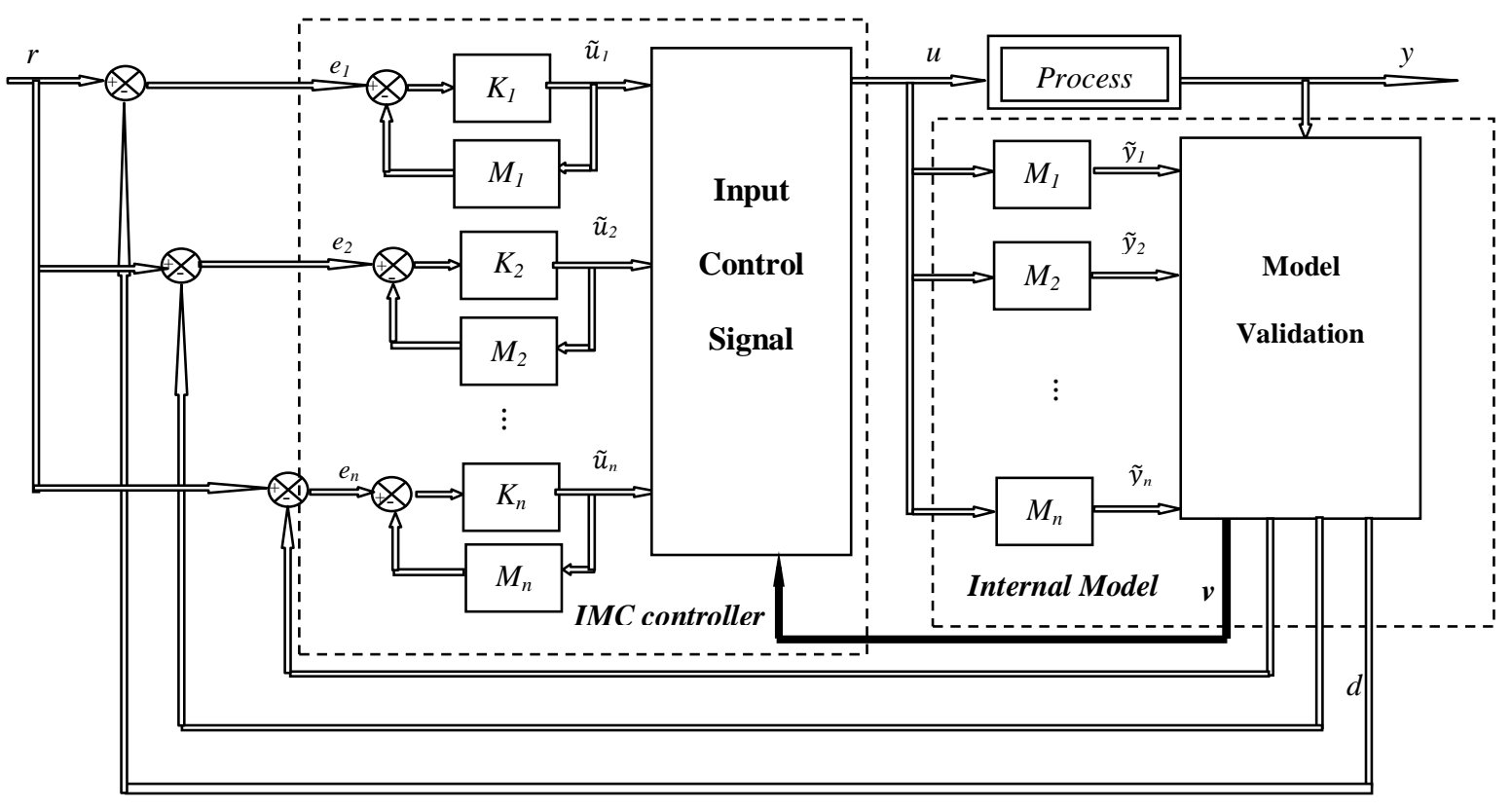

Fig.3. Proposed structure for internal multimodel control designed for nonlinear multivariable systems

For each model is associated a controller vector $\tilde{u}_{i}$ and an output vector $\tilde{y}_{i}$. The $M_{i}$ can be represented by $\left(A_{i}, B_{i}, C_{i}, D_{i}\right)$, where $A_{i}, B_{i}, C_{i}$ and $D_{i}$ are the state matrices.

\section{B. The switching method}

First of all, let's define the $n$ distance vectors $d_{i}, d_{i}=$ $\left\{d_{j i}\right\}_{j=1 \ldots m} . d_{i}$ characterizes the difference between system outputs $y$ and model outputs $\tilde{y}_{i}, y=\left\{y_{i}\right\}_{i=1 \ldots m}$ and $\tilde{y}_{i}=$ $\left\{\tilde{y}_{i j}\right\}_{j=1 \ldots m}$.

$$
d_{j i}(t)=y_{j}(t)-\tilde{y}_{i j}(t), i=1 \ldots n \text { and } j=1 \ldots m
$$

Let's consider $f_{i}$ such that

$$
f_{i}(t)=\sum_{j=1}^{m}\left\|d_{j i}(t)\right\|, i=1 \ldots n \text { and } j=1 \ldots m
$$

The controller correspondent to the model with the lowest $f_{i}$ is the one to apply to the system.

\section{The residues techniques}

For the residues techniques, all controllers participate on calculating the final controller applied to the system, according to their validities $v_{i}, i=1 \ldots n$.

To quantify the contribution of the $n$ partial command $\tilde{u}_{i}=\left\{\tilde{u}_{i j}\right\}_{j=1 \ldots m}$ to the global controller of the system, it's necessary to define validity values $v_{i}$, making it possible to give preemption, at every moment, to the nearest model at the detriment of the others.

$$
v_{i}=\frac{\frac{1}{\left\|f_{i}\right\|}}{\frac{1}{\sum_{i=1}^{m}\left\|f_{i}\right\|}}
$$

The control law $u_{i}$ to be applied to the system is given by the following expression.

$$
u_{i}(t)=\sum_{j=1}^{n} v_{j}(t) \tilde{u}_{i j}(t), i=1 \ldots m
$$

This control law depends on the partial commands and the validity coefficients relating to the models $M_{i}$.

\section{APPLICATION}

In order to illustrate the proposed internal multimodel control for multivariable nonlinear systems, let's consider the following multivariable system with two inputs two outputs $(m=2)$.

$$
\left\{\begin{array}{c}
\dot{x}_{1}(t)=-2 x_{1}(t)-\cos \left(x_{1}(t)\right) x_{2}(t)+u_{1}(t) \\
\dot{x_{2}}(t)=\cos \left(x_{1}(t)\right) x_{1}(t)-\left|x_{2}(t)\right| x_{2}(t)+5 u_{2}(t)
\end{array}\right.
$$

The plant equations can be transformed to the following state space equations

$$
\left\{\begin{array}{l}
\dot{x}(t)=A x(t)+B u(t) \\
y(t)=C x(t)+D u(t)
\end{array}\right.
$$

Where

$$
\begin{aligned}
& x(t)=\left(\begin{array}{l}
x_{1}(t) \\
x_{2}(t)
\end{array}\right), A=\left(\begin{array}{cc}
-2 & -\cos \left(x_{1}(t)\right) \\
\cos \left(x_{1}(t)\right) & -\left|x_{2}(t)\right|
\end{array}\right), \\
& B=\left(\begin{array}{ll}
1 & 0 \\
0 & 5
\end{array}\right), u(t)=\left(\begin{array}{l}
u_{1}(t) \\
u_{2}(t)
\end{array}\right), y=\left(\begin{array}{l}
y_{1}(t) \\
y_{2}(t)
\end{array}\right), \\
& C=\left(\begin{array}{ll}
1 & 0 \\
0 & 1
\end{array}\right) \text { and } D=0 .
\end{aligned}
$$

We propose to apply the convex polytopic transformation in order to define the models describing the system.

$$
\dot{x}=\sum_{i=1}^{n} \mu_{i}\left(A_{i} x+B u\right) \quad i=1 \ldots n
$$


$A_{i}, B, C$ and $D$ define the local models and the $\mu_{i}$ are the activation functions.

We suppose that the matrix $A=\left\{a_{i j}\right\}$ such us $a_{i j}$ are bounded

$$
a_{i j} \in\left[a_{i j \min }, a_{i j \max }\right], i, j=1,2
$$

Let $\cos \left(x_{1}(t)\right) \in[-1,1]$ and $x_{2} \in[0,10]$.

For the proposed system, $2^{2}$ models $\left(A_{i}, B, C, D\right)$ are proposed to describe the nonlinear multivariable system

$$
\begin{aligned}
A_{1} & =\left(\begin{array}{cc}
-2 & -1 \\
1 & -10
\end{array}\right), A_{2}=\left(\begin{array}{cc}
-2 & -1 \\
1 & 0
\end{array}\right) \\
A_{3} & =\left(\begin{array}{cc}
-2 & 1 \\
-1 & -10
\end{array}\right) \text { and } A_{4}=\left(\begin{array}{ll}
-2 & 1 \\
-1 & 0
\end{array}\right) .
\end{aligned}
$$

The considered local models for this example are significantly different from each others. This allows seeing clearly the difference between the two proposed methods applied to the system.

\section{A. Simulation results for the switching method}

Simulation results are shown in Fig. 4, 5 and 6 where the reference vector $\mathrm{r}$ is $r=\left(\begin{array}{l}1 \\ 1\end{array}\right)$, and the gain matrices $K_{i}$, $i=1 \ldots 4$ such that $K_{i}=\left(\begin{array}{cc}30 & 0 \\ 0 & 30\end{array}\right)$.

Fig. 4, 5 and 6 display simulation results for the switching method. Fig. 4 represents the internal multimodel controllers $u_{1}(t)$ and $u_{2}(t)$. System outputs $y_{1}(t)$ and $y_{2}(t)$ are displayed in Fig. 5. Control signals are elevated and varied from one moment to other. This is due to the switching between linear models leading to control signals variation. It can be shown a sluggish output responses (the system outputs reach the references after 80seconds) and an important overshoot. Fig. 6 displays the chosen models for the switching method. Their correspondent controllers are applied to the system. For this example, the switching method hasn't shown satisfactory results.

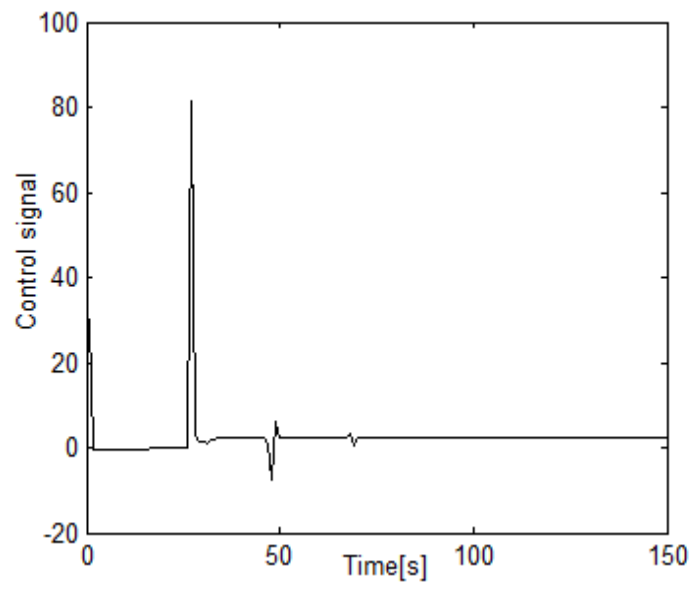

(a)

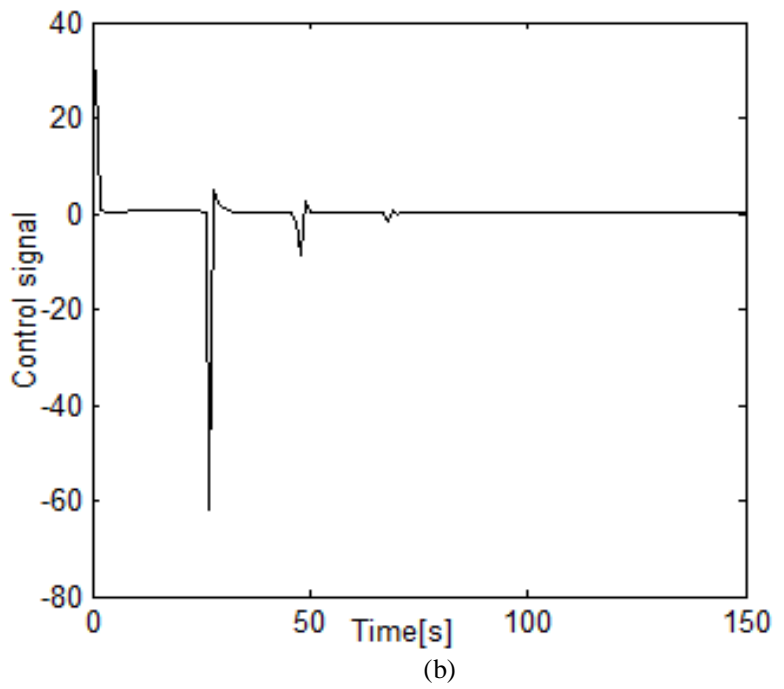

Fig.4. (a)Control signal $u_{1}$ (b) Control signal $u_{2}$

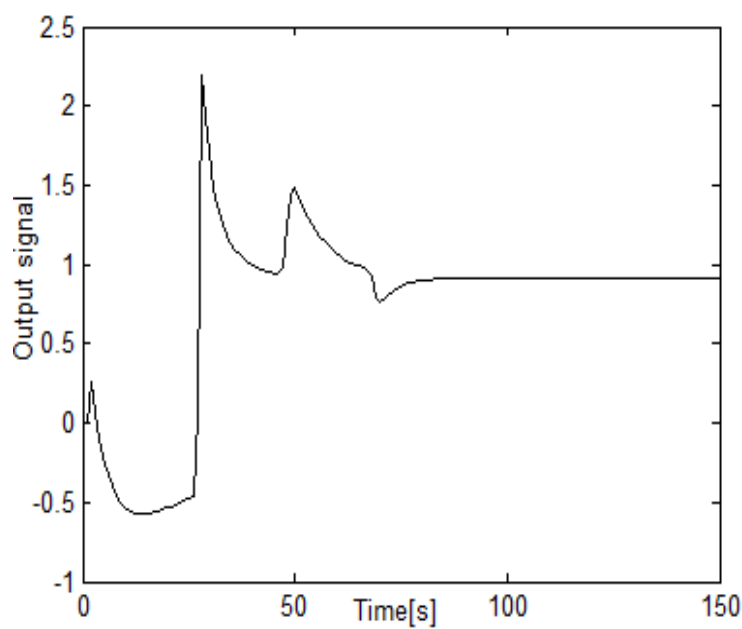

(a)

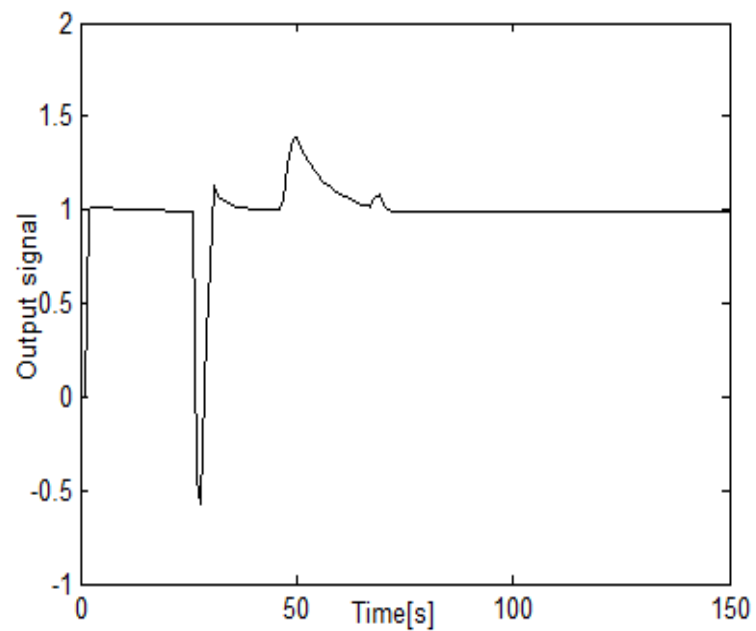

(b)

Fig.5. (a) Output signal $y_{1}$ (b) Output signal $y_{2}$ 


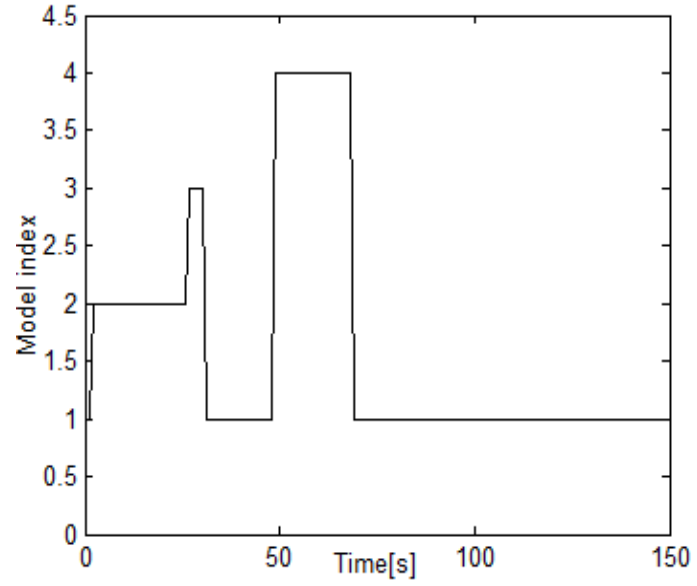

Fig.6. Chosen models for the switching method

\section{B. Simulation results for the residues techniques}

Simulation parameters for residues techniques are taken the same as the switching method. Fig. 7, 8 and 9 display simulation results for the residues techniques. Fig. 7 represents the evolution of the internal multimodel controllers $u_{1}(t)$ and $u_{2}(t)$. System outputs $y_{1}(t)$ and $y_{2}(t)$ are displayed in Fig. 8. The system outputs present an acceptable overshoot and are able to reach perfectly the references at the steady state. Fig. 9 shows model's validities.

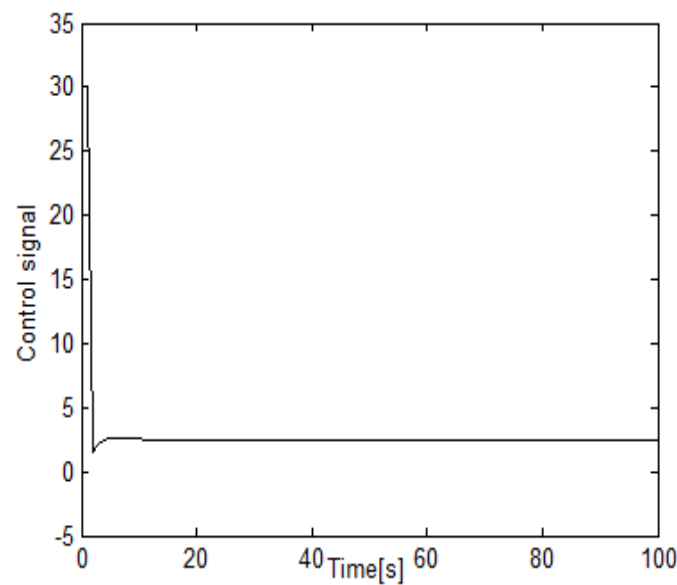

(a)

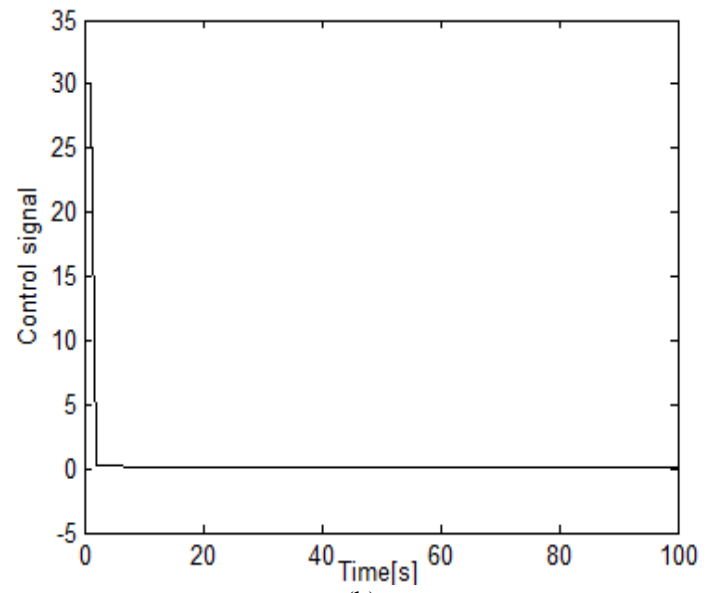

(b)
Fig.7. (A) Control signal $\mathrm{u}_{1}$ (b) Control signal $\mathrm{u}_{2}$

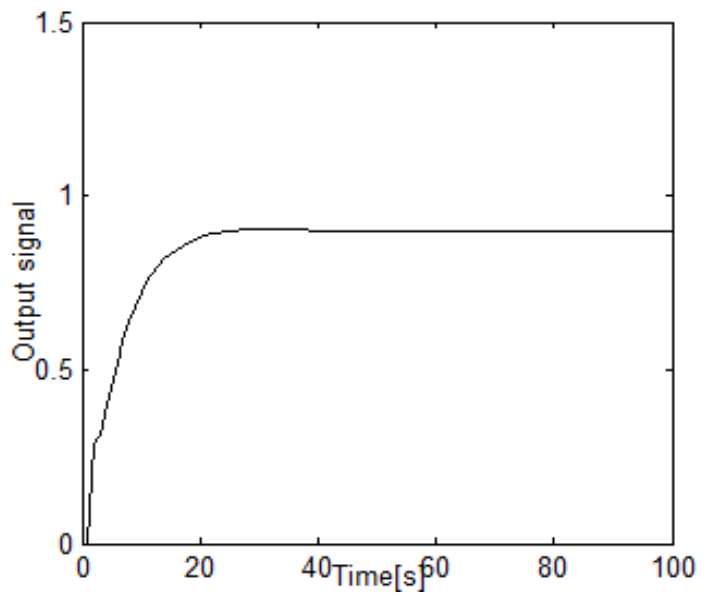

(a)

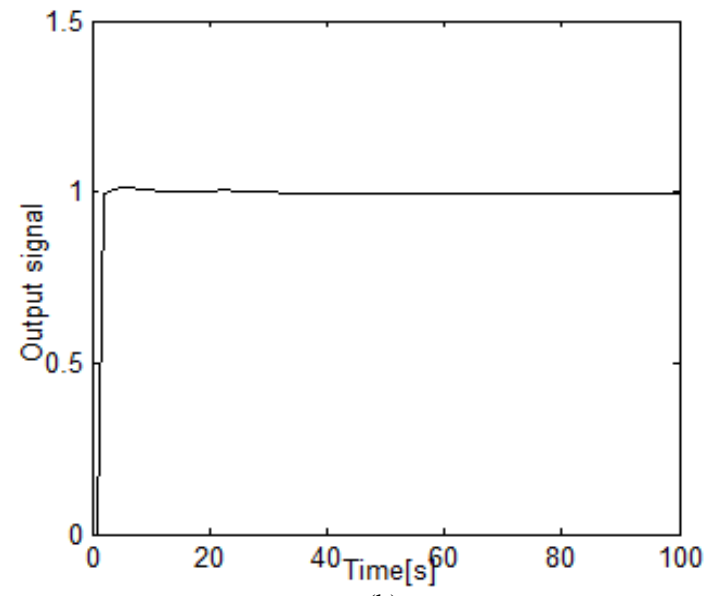

(b)

Fig.8. (a) Output signal $y_{1}$ (b) Output signal $y_{2}$

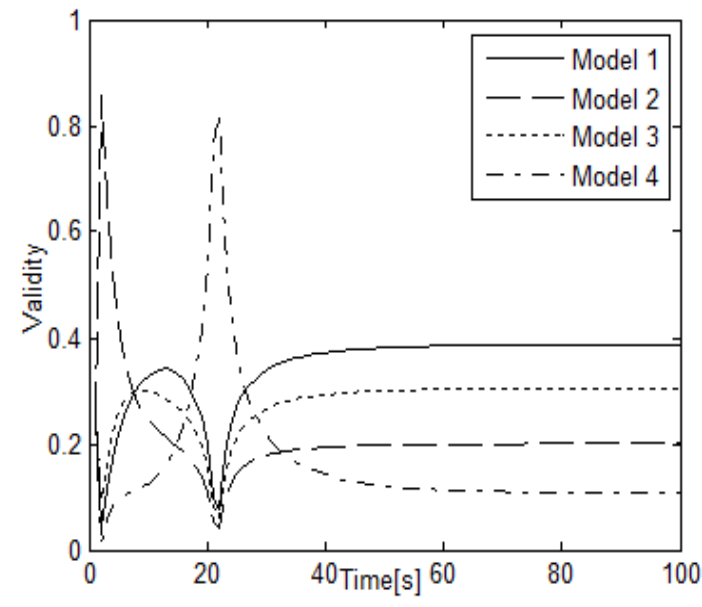

Fig.9. Validity indices

\section{CONCLUSION}

In this paper, two structures for internal multimodel control were proposed for nonlinear multivariable systems. 
The first structure is based on the determination of control signals by switching between different inverse models outputs. While the second one is based on using the residues techniques as fusion method to calculate the control signal.

The implementation of the second structure based on residues techniques is more complex and needs more computation time than the first one based on the switching method, but it is more efficient. In fact, by using the residues techniques, the system outputs reach quickly the imposed reference without important overshoot.

\section{REFERENCES}

[1] S. Bel Hadj Ali, "Commande par modèle interne de processus dynamiques", PhD, ENIT, Tunis, 2003.

[2] M. Benrejeb, M. Naceur, D. Soudani, "On an internal model controller based on the use of a specific inverse model", ACIDCA 2005, pp.623 626, Tozeur, 2005.

[3] N. Touati , D. Soudani., M. Naceur and M. Benrejeb , "On the internal model control of multivariable linear system", International Conference on Sciences and Techniques of Automatic control and computer engineering, STA'2011, Sousse, 2011.
[4] N. Touati, D. Soudani., M. Naceur and M. Benrejeb, "Internal Model Control of Coupled Tanks Process", 4th Conference of Applied Research and Technology Transfer CRATT'2012, Tunis, 2012.

[5] R. H. Nyström, J. M., Böling, J. M. Ramstedt, H. Toivonen and K. Häggblom, "Application of robust and multimodel control methods to an ill-conditioned distillation column", Original Research Article Journal of

[6] Process Control, Volume 12, Issue 1, January 2002, pp. 39-53.

[7] M. Morari and E. Zafiriou , "Robust Process Control”, Ed. Prentice Hall, Englewood cliffs, N.J., 1989.

[8] M. A. Henson and D. E. Seborg, "Internal Model Control Strategy for Nonlinear Systems”, A.I.Ch.E. Journal, vol. 37, 1991, pp. 1065-1081.

[9] J. Calvet and Y. Arkun, "Feedforward and feedback linearization of non-linear systems and its implementation using internal model control," Ind. Eng. Chem. Res., vol. 27, no. 10, pp. 327*346, 1988.

[10] C.G. Economou, M. Morari, and B. O. Palsson, "Internal model control 5. Extension to nonlinear systems,'”nd. Eng. Chem. Proc. Des. and Dev., vol. 25, pp. 403-409, 1986.

[11] C. E. Garcia and M. Morari,"Internal model control. 1. a unifying review and some results', Industrial Engineering Chemistry Process Design and Development", vol. 21, 1982, pp. 403-411. 